\title{
The generation of two isochronous sequences in parallel
}

\author{
DIANA DEUTSCH \\ University of California, San Diego, La Jolla, California
}

\begin{abstract}
Subjects were presented with two parallel pulse trains through earphones, one to each ear. The pulse trains were isochronous, and the durations of the intervals associated with the right and left trains were systematically varied, so as to give rise to both simple rhythms and poly. rhythms. The subjects were required to tap with the right hand in synchrony with the train delivered to the right ear, and to tap with the left hand in synchrony with the train delivered to the left ear. Accuracy of performance in polyrhythm contexts was substantially lower than in simple rhythm contexts, and decreased with an increase in the complexity of the associated polyrhythm. It was concluded that the performer develops a representation of the pattern as an integrated whole, and that performance accuracy is inversely related to pattern complexity.
\end{abstract}

An interesting but little investigated area of research concerns the generation of temporally patterned sequences. Performance levels on such tasks vary substantially depending on the type of pattern employed. Thus, isochronous sequences have been found to be generated to a high degree of accuracy (Allan, 1979; Bartlett \& Bartlett, 1959; Michon, 1967; Treisman, 1963; Wagner, 1971; Wing \& Kristofferson, 1973a, 1973b). In contrast, the task of generating irregular sequences has been found to be very difficult, with subjects tending to generate interresponse intervals that were approximately identical or that stood in a ratio of approximately $2: 1$ (Fraisse, 1982). A tendency toward simplification of temporal relationships was also observed by Montpellier (1935). He presented subjects with a set of targets which they were required to tap in rapid succession, and found that intertap intervals tended either toward equality or toward ratios of $2: 1$ or 3:1. Povel (1981) studied the imitation of repeating temporal patterns that consisted of two or more intervals standing in various ratios to each other. He obtained substantial differences in performance levels depending on these ratios. For simple patterns, those with intervals standing in a ratio of $2: 1$ were accurately imitated, and those standing in other ratios were imitated less well, with systematic deviations in performance tending toward a ratio of $2: 1$. For more complex patterns, those that could be represented in terms of a simple hierarchical structure were well imitated, and those that could not be were imitated only poorly. The existing body of evidence suggests, therefore,

This work was supported by United States Public Health Service Grant MH-21001. I am grateful to Robert Welker for help in the training phase of the experiment, and to Greg Stone for performing the statistical analyses. Requests for reprints should be sent to Diana Deutsch, Department of Psychology, C-009, University of California at San Diego, La Jolla, California 92093. that temporal patterns are encoded by the observer in hierarchical form, and that such hierarchies are invoked for their generation. (See, also, Cooper \& Meyer, 1960; Martin, 1972; Perkins, 1974; Sturges \& Martin, 1974; Summers, 1975; Westergaard, 1975; Yeston, 1976.)

The present article is addressed to the question of how different temporal patterns are generated in parallel. The simplest case is explored, that is, that of two isochronous sequences. We may ask whether these may be generated in parallel independently, or whether this task requires their integration into a single internal representation. From one point of view, since the dual task is descriptively very simple, we might expect that parallel processing would readily occur. However, the experimental findings on the generation of single patterns lead to the alternative prediction that accuracy in producing parallel patterns would also depend on the temporal relationships involved. Observations outside the laboratory support this assumption. In playing keyboard instruments, it is easy to generate two isochronous sequences in parallel, one with each hand, provided that the interval associated with one sequence is an integral multiple of the interval associated with the other sequence. However, when this relationship does not hold (as in the case of polyrhythms), performance is surprisingly difficult. In order to achieve accuracy, the performer generally learns first to produce the integrated pattern, and then to associate each component of the pattern with the appropriate hand, so that the two isochronous sequences finally emerge in parallel.

Experimental findings on the concurrent performance of two motor tasks provide further evidence that some form of temporal integration in involved. Peters (1977) asked subjects to beat out a rhythm while simultaneously reciting a nursery rhyme with 
proper rhythmic intonation. No subject was able to perform this task, and it was concluded that the nervous system, in the voluntary guidance of movement, can produce only one basic rhythm at a time. Klapp (1979) found that subjects were well able to perform two periodic keypresses simultaneously, one with each hand, when these occurred at identical rates, or when the rate for the left hand was twice that for the right. However, when the left-hand rate differed slightly from the right-hand rate, or when the right hand was pressing as rapidly as possible, performance was considerably poorer. Furthermore, Kelso, Southard, and Goodman (1979) asked subjects to make concurrent movements with each hand to targets that differed in size and in distance from the resting position. Under these conditions, the hands appeared constrained to move in synchrony, so that they arrived at their respective targets at the same time. These three studies therefore argue that there are strong temporal constraints on the performance of concurrent actions, as though these were controlled by a single timing mechanism (see, also, Keele, 1981).

In contrast, with highly skilled performance, two differently timed motor sequences may be successfully generated in parallel (Shaffer, 1981). For example, pianists are able to perform polyrhythms with accuracy following extensive training. One possible explanation of this finding is that the performer develops the ability to utilize independent timing mechanisms. Alternatively, we may propose that the performer develops a representation of the patterns as an integrated whole (see, also, Beauvillain \& Fraisse, in press). These two hypotheses lead to different predictions. If independent timing mechanisms were utilized, then success in generating two isochronous sequences in parallel should not depend on the temporal relationships between them. However, if performance levels depended on the development of an integrated representation, then accuracy in performance should be related to the structural simplicity of the integrated pattern.

For illustration, we can take the 3-against-2 polyrhythm. At $1200 \mathrm{msec}$ per pattern repetition, this may be described as consisting of a string of 400 msec intervals together with a string of 600 -msec intervals. Alternatively, the pattern may be described in terms of a hierarchy such as that shown in Figure 1a, in which the 1,200-msec time span is divided into three 400-msec segments, which are each divided into two 200 -msec segments. The pattern $(R / L$ R L R -), when associated with the lowest level structure, then produces a 3 -against-2 polyrhythm. This model is described in detail in Deutsch and Feroe (Note 1).

If polyrhythms are internally represented in such a fashion, then we would expect that ease in producing such rhythms would depend on the complexity
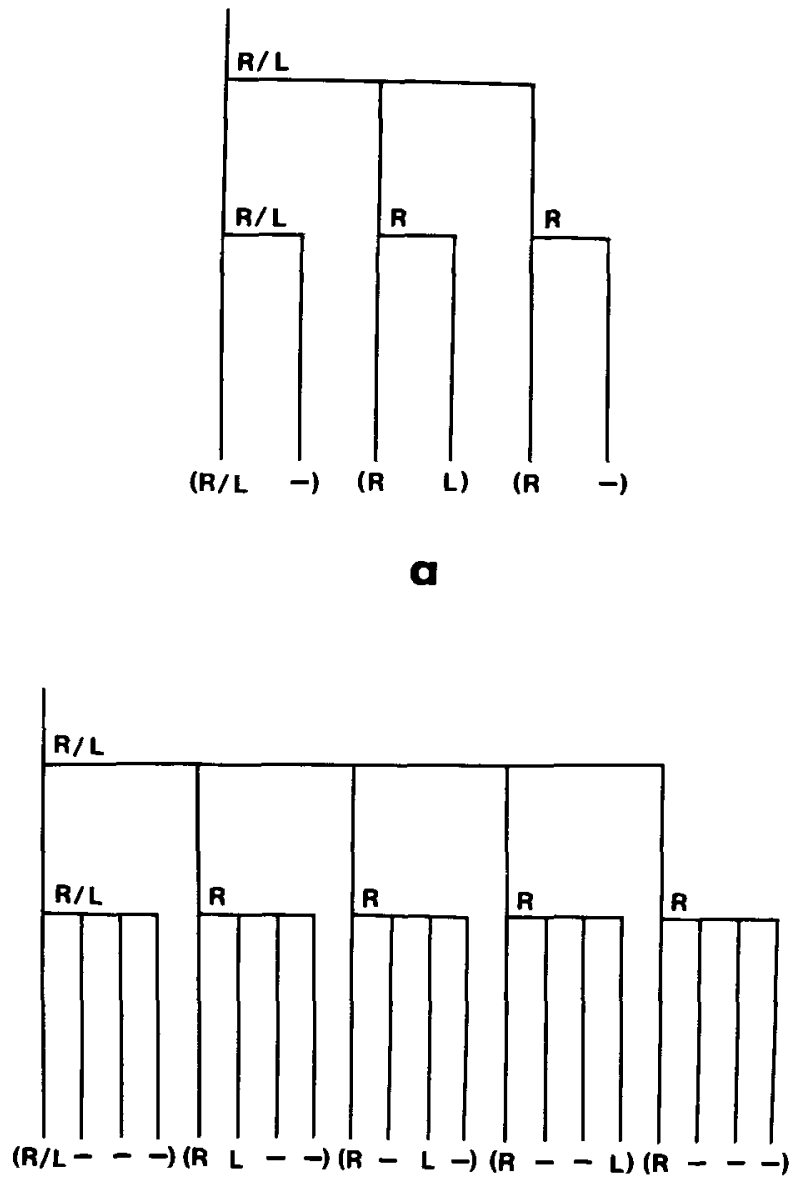

b

Figure 1. Hierarchical representation of polyrhythms. It is assumed that the time span corresponding to one cycle of a polyrhythm is divided into equal segments associated with one train of events. These segments are themselves divided into equal segments, enabling the representation of the other train of events. (a) Representation of the 3-against-2 polyrhythm. (b) Representation of the 5-against -4 polyrhythm. $R=$ right-hand tap, $L=$ lefthand $\operatorname{tap}, R / L=$ right- and left-hand taps, - = no tap.

of the associated representation. Thus, for example, the 5 -against- 4 polyrhythm would have a representation such as that shown in Figure 1b. It can be seen that this involves a pattern that is considerably more complex than that for the 3-against-2 polyrhythm. The 5-against-4 polyrhythm should therefore be produced considerably less accurately than the 3-against2 polyrhythm.

The present experiment was designed to test this hypothesis. Subjects were presented with two parallel pulse trains through earphones, one to each ear. They were required to tap with the right hand in synchrony with the train delivered to the right ear, and to tap with the left hand in synchrony with the train delivered to the left ear. ${ }^{1}$ The pulse trains were isochronous, and the durations of the intervals asso- 
ciated with the right and left trains were systematically varied, so as to give rise to both simple rhythms and polyrhythms. It was hypothesized that tapping accuracy would decrease with an increase in the complexity of the associated pattern description.

\section{METHOD}

\section{Stimuli and Conditions}

The stimuli consisted of trains of 50 -msec sine-wave tones (or blips) presented through headphones at a level of $75 \mathrm{~dB}$ SPL. The frequencies of the blips presented to the right and left ears were 1100 and $800 \mathrm{~Hz}$, respectively. (A frequency difference between the blips was necessary to prevent the impression of a single blip in the center of the head when these were presented simultaneously to both ears.) The subjects were instructed to tap with the right forefinger in synchrony with the blips delivered to the right ear, and with the left forefinger in synchrony with the blips delivered to the left ear.

The experimental conditions were designed as follows. A 1,200msec interval between blip onsets served as the base interval. This was divided by 1 , producing a 1,200 -msec onset-to-onset interval; by 2 , producing a 600 -msec onset-to-onset interval; by 3 , producing a 400-msec onset-to-onset interval; by 4 , producing a 300 -msec onset-to-onset interval; and by 5 , producing a 240 -msec onset-toonset interval. These intervals were chosen because they are in the range in which the following of pulse trains most readily occurs (Fraisse, 1982; Handel \& Oshinsky, 1981). ${ }^{2}$ Each of these intervals presented to the right ear was paired equally often with each of these intervals presented to the left ear, producing 25 conditions in all. The first blips of each train always occurred in synchrony, so that the blips were synchronous at least once every $1,200 \mathrm{msec}$. Thus, the following simple rhythms were generated: 1 against 1; 2 against $1 ; 3$ against $1 ; 4$ against 1 ; and 5 against 1 . Also, the following polyrhythms were generated: 3 against 2; 5 against 2 , 4 against 3,5 against 3 , and 5 against 4 .

\section{Apparatus}

Tones were generated by two Wavetek function generators (Model 155) controlled by a PDP-11/03 computer. The output was passed through a Crown amplifier, and was presented to the subject through matched headphones (Grason-Stadler Model TDH-49) in a sound-insulated booth. The subject responded by tapping on two $3 \times 1$ in. metal plates mounted on a box. He or she tapped with the right forefinger on the plate to the right and with the left forefinger on the plate to the left. A third $3 \times 1$ in. metal plate was centered between these two, and the subject tapped this plate to initiate a run of trials. A red LED was mounted above the plates.

\section{Procedure}

Each subject was tested individually. To initiate a run of trials, the LED flashed on and off for $5 \mathrm{sec}$, following which the subject tapped the center plate to indicate his/her readiness. Five seconds later, one of the conditions was presented for $52 \mathrm{sec}$. The subjects tapped with the right forefinger in synchrony with the blips presented to the right ear and with the left forefinger in synchrony with the blips presented to the left ear. The first $12 \mathrm{sec}$ were treated as a warm-up period. For the remaining $\mathbf{4 0} \mathrm{sec}$, the LED was turned on and the taps were recorded. At the termination of the trial, the LED was turned off, and there followed a 10-sec delay. The next trial then began, and a different condition was presented. At the termination of a run of five trials, the subject was given a rest period of approximately $2 \mathrm{~min}$.

Each experimental session consisted of five runs of five trials each, so that each of the 25 conditions was presented once during a session. The order of presentation of the conditions within a session varied randomly. Each subject participated for six experimental sessions.

\section{Subjects}

Three musically trained subjects participated in the experiments. None had received special training in the production of polyrhythms. All subjects were given several practice sessions before the experiment began.

\section{RESULTS}

The measure of tapping accuracy was variability in temporal position of taps relative to blips, determined for each channel separately. This was computed as follows. Each tap was associated with the blip that was closest in time on the corresponding channel, and the signed timed difference between the two was determined. The standard deviation of these signed time differences was then computed for each trial, for each channel separately.

Since the two hands did not differ significantly in overall tapping accuracy $(F<1)$, the data for the two hands were pooled. Accuracy in tapping at each rate was then plotted as a function of the rate at which the other hand was tapping. Figures 2 to 6 display,

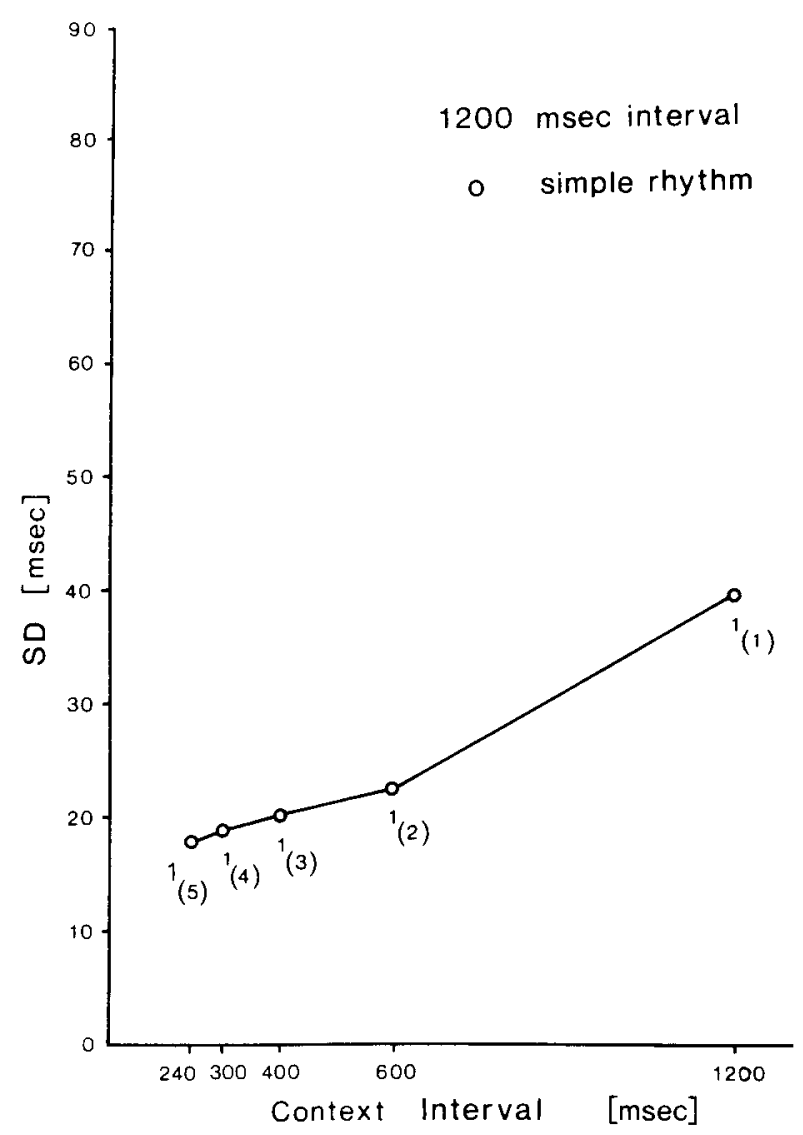

Figure 2. Performance accuracy for hand tapping at a 1,200msec rate, plotted against tapping rate of the other hand. Numerals inside display indicate rhythm being executed. Thus, $\mathbf{1}_{(5)}$ indicates a 5-against-1 rhythm, with aceuracy plotted for the slower component. $1_{(1)}$ indicates the two hands tupping in synchrony at the 1,200-msec rate. 
for each tapping rate, the average standard deviations obtained at each context tapping rate. The effect of context rate was highly significant at all tapping rates $[F(4,8)=25.61, p<.001$, for the 1,200 msec tapping rate; $F(4,8)=8.16, p<.01$, for the 600 msec tapping rate; $F(4,8)=42.13, p<.001$, for the 400-msec tapping rate; $F(4,8)=38.87, p<.001$, for the 300-msec tapping rate; $F(4,8)=38.35, p<.001$, for the 240-msec tapping rate].

At the 1,200 -msec tapping rate, only simple rhythms were executed. As can be seen from Figure 2, accuracy was a monotonically increasing function of context rate. As described above, this effect was highly significant.

At the 600-msec tapping rate, there were three context rates that resulted in simple rhythms $\left(2_{(1)}, 2_{(2)}\right.$, and $\left.2_{(4)}\right)$. As shown in Figure 3, overall accuracy was very high for the simple rhythms, and increased slightly with an increase in context rate. This effect was significant $[F(2,4)=13.11, p<.05]$. Furthermore, accuracy was significantly lower in polyrhythm contexts than in simple rhythm contexts $\left(2_{(3)}, 3_{(5)}\right.$ vs. $2_{(1)}$,

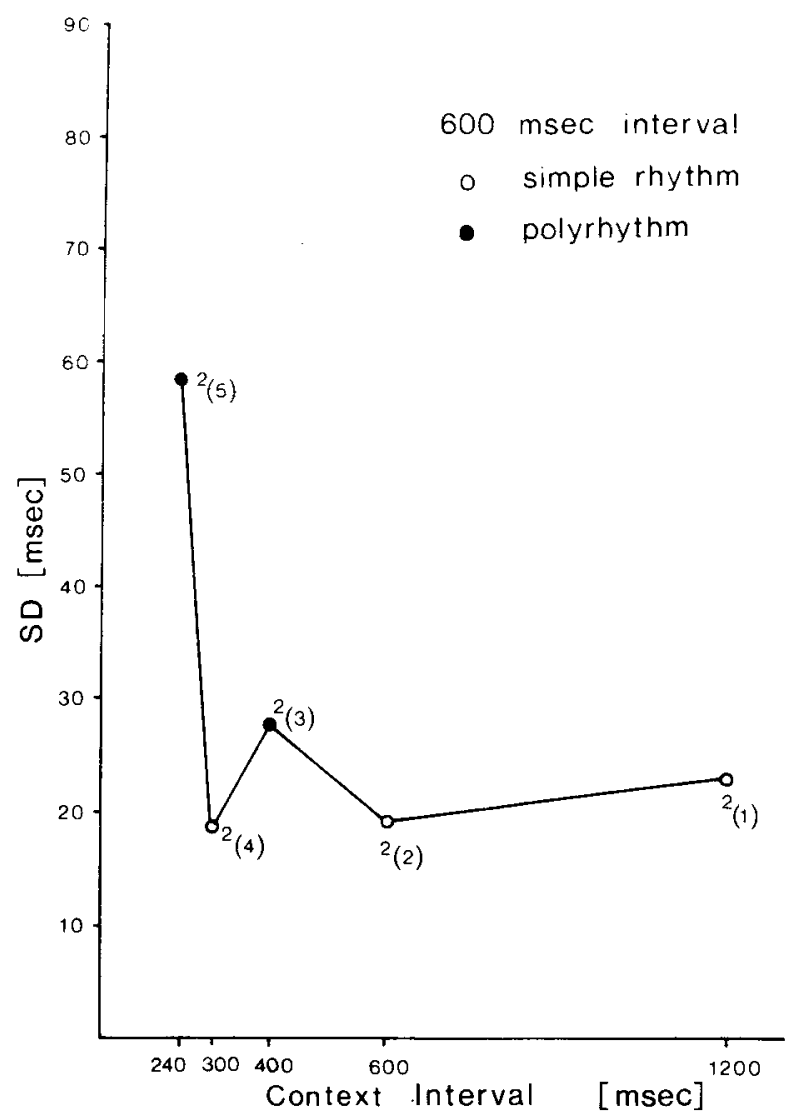

Figure 3. Performance accuracy for hand tapping at a $600-\mathrm{msec}$ rate, plotted against tapping rate of the other hand. Thus, $\mathbf{2}_{\mathbf{3}}$ ) indicates a 3-against-2 polyrhythm, with accuracy plotted for the slower component. Note that $2_{(1)}$ and $2_{(4)}$ both indicate a 2-against-1 rhythm, but executed at different speeds. $2_{(2)}$ indicates the two hands tapping in synchrony at the 600-msec rate.
$2_{(2)}, 2_{(4)} ;$ observed difference $=23.0, \mathrm{CR}_{\mathrm{LSD}}=17.8$, $\mathrm{p}<.01){ }^{3}$ Significant differences also emerged between the different polyrhythm contexts $\left(2_{(3)}\right.$ vs. $2_{(3)}$; observed difference $\left.=30.2, \mathrm{CR}_{\mathrm{LSD}}=19, \mathrm{p}<.01\right)$.

At the 400-msec tapping rate, there were two context rates that resulted in simple rhythms $\left(3_{(1)}\right.$ and $\left.3_{(3)}\right)$ and three that resulted in polyrhythms $\left(3_{(2)}, 3_{(4)}\right.$, and $\left.3_{(5)}\right)$. As shown in Figure 4, accuracy was very high in simple rhythm contexts, and higher at the higher context rate than at the lower. (This difference, however, was not significant.) Overall accuracy was significantly lower in polyrhythm contexts than in simple rhythm contexts $\left(3_{(2)}, 3_{(4)}, 3_{(5)}\right.$ vs. $3_{(1)}, 3_{(3)}$; observed difference $\left.=49.4, \mathrm{CR}_{\mathrm{LSD}}=17.7, \mathrm{p}<.01\right)$. Significant differences also emerged between the different polyrhythm contexts $\left(3_{(2)}\right.$ vs. $3_{(4)}$; observed difference $=63.9, \mathrm{CR}_{\mathrm{LSD}}=27.5, \mathrm{p}<.01 ; 3_{(2)}$ vs. $3_{(5)}$; observed difference $=63.3, \mathrm{CR}_{\mathrm{LSD}}=27.5, \mathrm{p}<.01$ ). Since performance in conditions $3_{(4)}$ and $3_{(5)}$ was at chance, there was essentially no difference between these conditions.

At the 300-msec tapping rate, there were three context rates that resulted in simple rhythms $\left(4_{(1)}, 4_{(2)}\right.$, and $\left.4_{(4)}\right)$ and two that resulted in polyrhythms $\left(4_{(3)}\right.$ and $\left.4_{(3)}\right)$. As shown in Figure 5, overall accuracy was very high in simple rhythm contexts, and increased slightly with an increase in context rate. (This increase, however, was not significant.) Overall accuracy in polyrhythm contexts was significantly lower than in simple rhythm contexts $\left(4_{(3)}, 4_{(5)}\right.$ vs. $4_{(1)}, 4_{(2)}, 4_{(4)}$; observed difference $\left.=55.6, \mathrm{CR}_{\mathrm{LSD}}=15.1, \mathrm{p}<.01\right)$. Accuracy was higher for context rate $4_{(3)}$ than for context rate $\mathbf{4}_{(s)}$. However, since performance levels here approached chance, this difference was only marginally significant (observed difference $=13.6, \mathrm{CR}_{\mathrm{LSD}}=13$, $\mathrm{p}=.1$ )

At the 240-msec tapping rate, there were two context rates that resulted in simple rhythms $\left(5_{(1)}\right.$ and $5_{(5)}$ and three that resulted in polyrhythms $\left(5_{(2)}, 5_{(3)}\right.$, and $\left.5_{(4)}\right)$. As shown in Figure 6, overall accuracy was very high in simple rhythm contexts and slightly higher at the higher context rate than the lower. (This difference was not, however, statistically significant.) Overall accuracy was significantly lower in polyrhythm contexts than in simple rhythm contexts $\left(5_{(2)}, 5_{(3)}, 5_{(4)}\right.$ vs. $5_{(1)}, 5_{(5)}$; observed difference $=44.8, \mathrm{CR}_{\mathrm{LSD}}=12.9$, $\mathrm{p}<.01)$. Significant differences also emerged between the different polyrhythm contexts $\left(5_{(2)}\right.$ vs. $5_{(3)}$; observed difference $=22.2, \mathrm{CR}_{\mathrm{LSD}}=20, \mathrm{p}<.01$; $S_{(2)}$ vs. $S_{(4)}$; observed difference $=21.8, C R_{L S D}=20$, $\mathrm{p}<.01)$. Since performance in conditions $5_{(3)}$ and $5_{(4)}$ was at chance, there was essentially no difference between these conditions.

\section{DISCUSSION}

Three general findings have emerged from the present study. First, performance levels in simple 


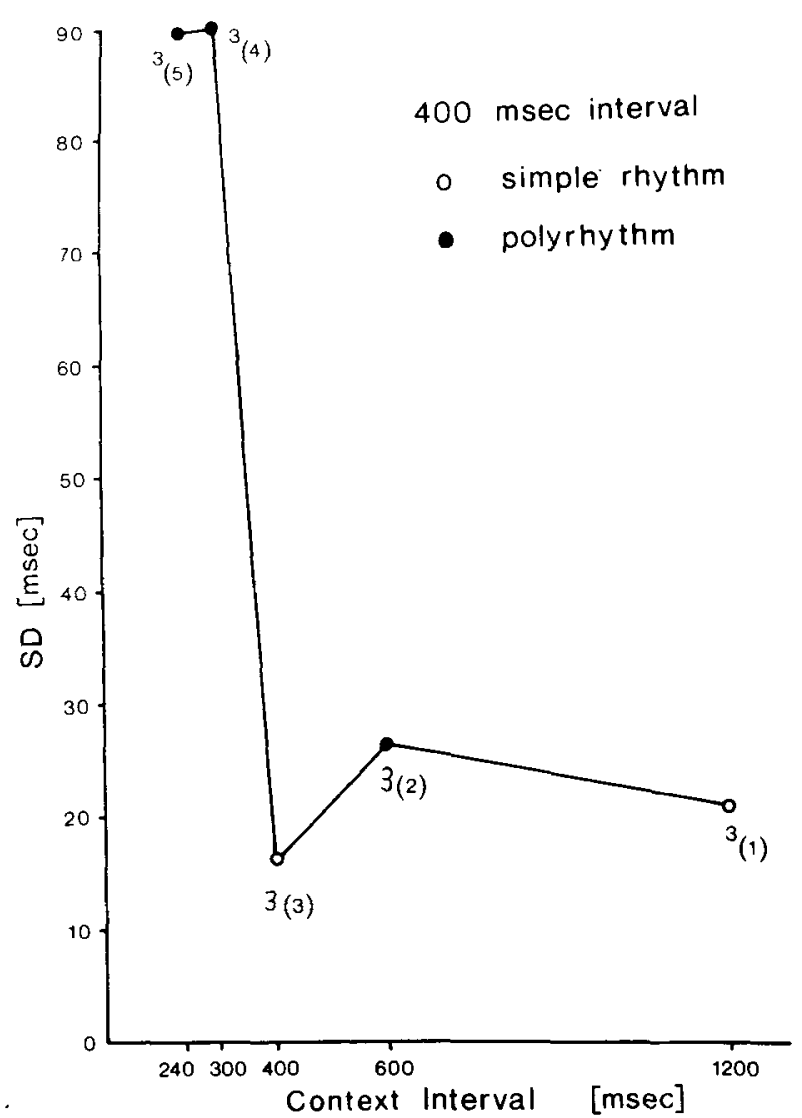

Figure 4. Performance accuracy for hand tapping at a 400-msec rate, plotted against tapping rate of the other hand. Thus, $3_{\text {(2) }}$ indicates 3-against-2 polyrbythm, with accurncy plotted for the faster component. $3_{(3)}$ indicates the two hands tapping in synchrony at the 400-msec rate.

rhythm contexts were very high, and they increased with an increase in context rate. Second, performance levels in polyrhythm contexts were substantially lower than in simple rhythm contexts. Third, there were substantial differences in performance levels between the different polyrhythm contexts.

The results from simple rhythm contexts are in accordance with those of other studies showing that accuracy of perception and production of temporal intervals increases with a decrease in the duration of these intervals (Allan, 1979; Bartlett \& Bartlett, 1959; Michon, 1967; Treisman, 1963; Wagner, 1971; Wing \& Kristofferson, 1973a, 1973b). If the context blips were used as cues to subdivide the 1,200 -msec time interval into smaller intervals, then accuracy should indeed increase as the durations of the context intervals decrease. It is interesting to note that the requirement to execute a larger number of taps per unit time with the context hand did not impair accuracy; rather, accuracy increased with an increase in the total number of taps to be executed. This implies that differences in accuracy were not much related to purely motor factors. It is also interesting to note that the simple rhythm involving division of the base interval into five segments ( 5 against 1 ) was very well executed. Western tonal music rarely involves such a division, so one might have expected performance here to be poorer than for the other simple rhythms. However, no such difficulty was apparent.

The results from polyrhythm contexts are in accordance with the hypothesis that accuracy in generating two sequences in parallel depends on the development of an integrated pattern representation. The present finding that accuracy in tapping at a given rate depends strongly on context rate cannot be explained by the hypothesis of independent timing mechanisms. Rather, the data are well explained on the hypothesis that the performer develops a representation of the pattern as an integrated whole, and that accuracy is inversely related to pattern complexity. Table 1 displays the description of each polyrhythm in terms of the sequence of events required to produce it. Taking as our measure of complexity the number of events contained in the pattern description, we find that accuracy at each rate was inversely

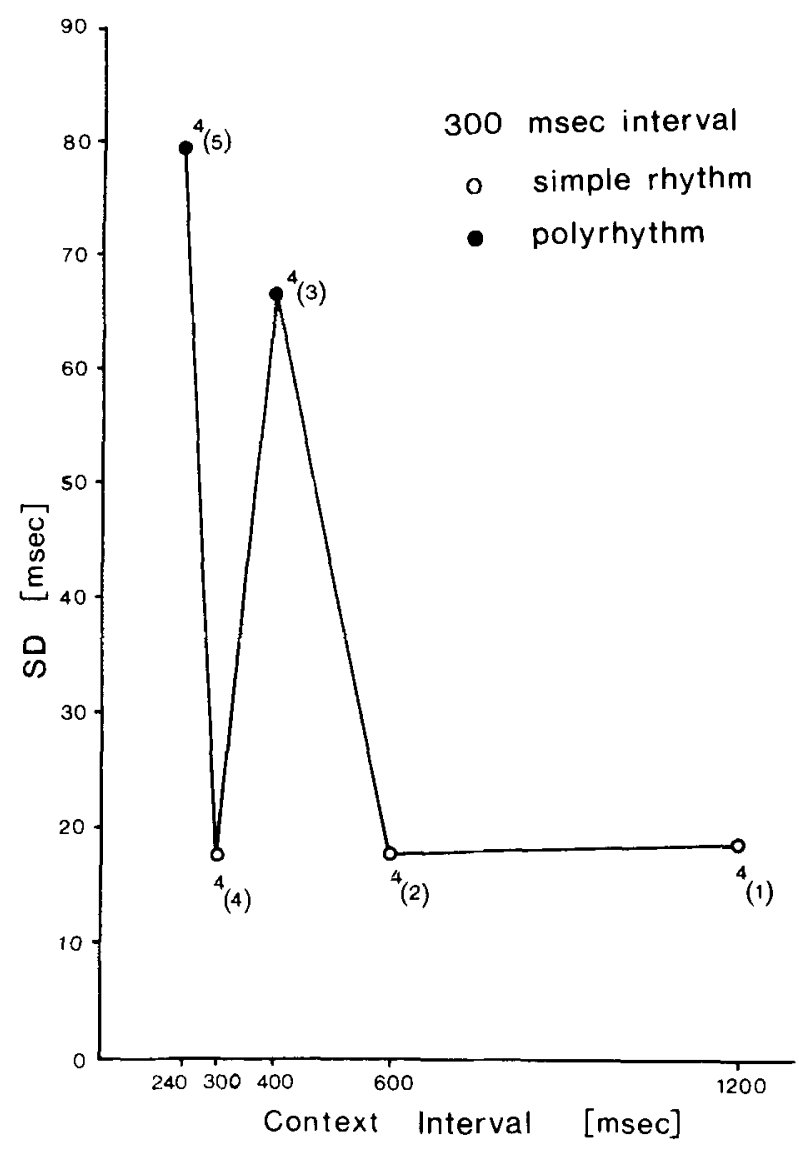

Figure 5. Performance accuracy for hand tapping at the 300msec rate, plotted against tapping rate of the other hand. Thus, 4(3) indicates a 4-againat-3 polyrhythm, whth accuracy plotted for the faster component. $4_{(2)}$ indicates a 2 -against-1 rhythm. $4_{(4)}$ indicates the two hands tapping in synchrony at the 300-msec rate. 


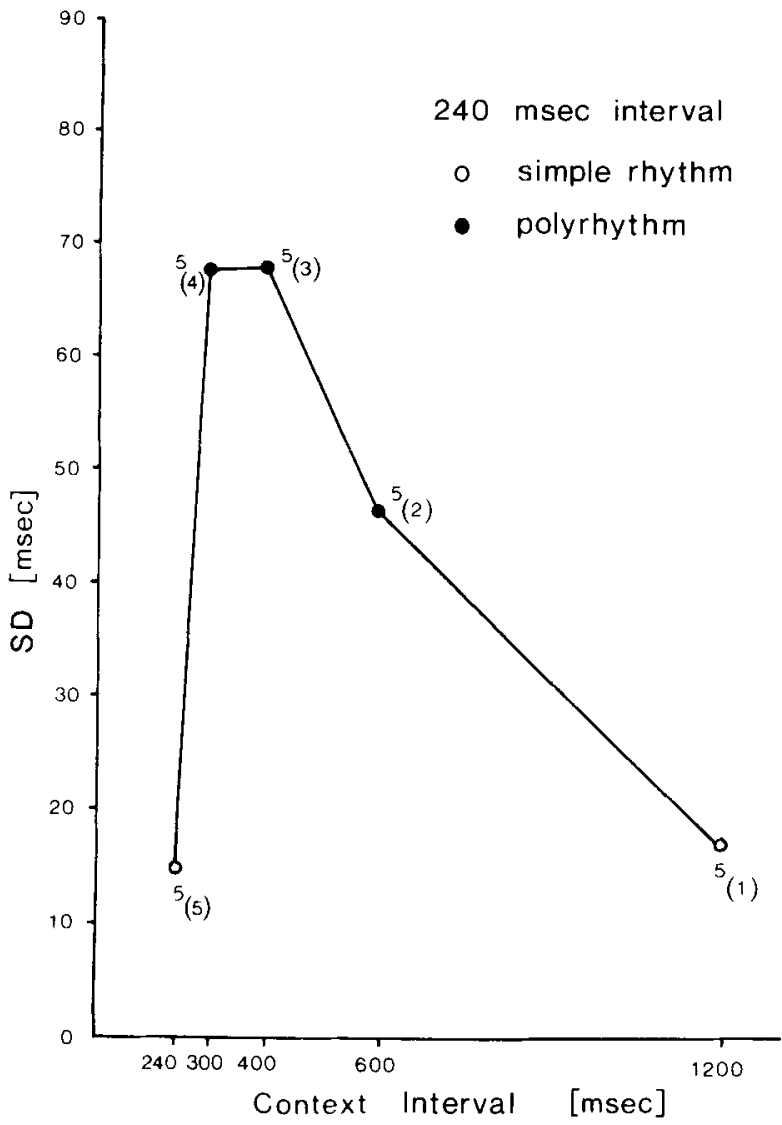

Figure 6. Performance accuracy for hand tapping at a 240 -msec rate, plotted against tapping rate of other hand. Thus, $5_{(2)}$ indicates a 5-against-2 polyrhythm, with accuracy plotted for the faster component. $5_{(5)}$ indicates the two hands tapping in synchrony at the 240-msec rate.

related to complexity of the polyrhythm being executed. Thus, at the $600-\mathrm{msec}$ tapping rate, accuracy was higher in the context of the 3-against-2 polyrhythm than in the context of the 5-against-2 polyrhythm. At the 400-msec tapping rate, accuracy was higher in the context of the 3-against-2 polyrhythm than in that of the 4-against-3 polyrhythm, and also higher in the context of the 3-against-2 polyrhythm than in that of the 5-against-3 polyrhythm. At the 300-msec tapping rate, accuracy was higher in the

Table 1

Polyrhythm Descriptions

\begin{tabular}{|c|c|}
\hline Polyrhythm & Taps \\
\hline $\begin{array}{l}\text { 3-against-2 } \\
\text { 5-against-2 } \\
\text { 4-against-3 } \\
\text { 5-against-3 } \\
\text { 5-against-4 }\end{array}$ & 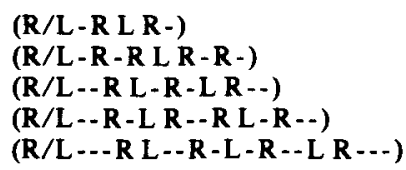 \\
\hline
\end{tabular}

Note- $R=$ right-hand tap, $L=$ left-hand tap, $R / L=$ right- and left-hand taps, - = no tap. context of the 4-against-3 polyrhythm than in that of the 5-against-4 polyrhythm. At the $240-\mathrm{msec}$ tapping rate, accuracy was higher in the context of the 5against-2 polyrhythm than in that of the 5-against3 polyrhythm, and also higher in the context of the 5 -against-2 polyrhythm than in that of the 5-against -4 polyrhythm. (The only comparisons that did not reflect this rank ordering of complexity were at the 400 msec tapping rate in the context of the 4-against-3 polyrhythm as compared with the 5-against-3 polyrhythm, and at the 240-msec tapping rate in the context of the 5-against-3 polyrhythm as compared with the 5-against-4 polyrhythm. In both these cases, performance levels were at chance, so that there were essentially no differences depending on context rate.)

The present study does not directly address the question of whether a timing hierarchy is involved in the pattern representation; indeed, the results are also consistent with the view of an internal representation that is nonhierarchical in nature. However, others have provided arguments for a hierarchical encoding of rhythmic structures (Cooper \& Meyer, 1960; Fraisse, 1982; Martin, 1972; Perkins, 1974; Povel, 1981; Sturges \& Martin, 1974; Summers, 1975; Westergaard, 1975; Yeston, 1976). However, if we take as our measure of pattern complexity the number of chunks required for a hierarchical pattern representation, together with the number of elements in each chunk, the same rank-ordering of complexity emerges. Thus, as shown in Figure 1, the representation for the 3-against-2 polyrhythm consists of one three-element higher level chunk (R/L R R) and three two-element lower level chunks (R/L-) $(R L)$ and (R-). In contrast, the 5-against-4 polyrhythm consists of one five-element higher level chunk (R/L R R R R) and five four-element lower level chunks (R/L---) (R L--) (R-L-) (R--L) and (R---). (See Deutsch \& Feroe, Note 1, for a formal description of this model.) The present results are therefore consistent with the view that the encoding of polyrhythms involves hierarchies of such a nature, and that performance levels in producing such polyrhythms are inversely related to the complexity of their associated hierarchies.

\section{REFERENCE NOTE}

1. Deutsch, D., \& Feroe, J. The internal representation of rhythmic patterns. Manuscript in preparation, 1983.

\section{REFERENCES}

Allan, L. G. The perception of time. Perception \& Psychophysics, 1979, 26, 340-354.

Bartlett, N. R., \& Bantlett, S. C. Synchronization of a motor response with an anticipated sensory event. Psychological Review, 1959, 66, 203-218.

Beauvillain, C., \& Fraisse, P. On the temporal control of polyrhythmic performance. Music Perception, in press.

COOPER, G. W., \& MEYER, L. B. The rhythmic structure of music. Chicago: University of Chicago Press, 1960. 
Fraisse, P. Rhythm and tempo. In D. Deutsch (Ed.), The psychology of music. New York: Academic Press, 1982.

HANDEL, S., \& Oshinsky, J. S. The meter of syncopated auditory polyrhythms. Perception \& Psychophysics, 1981, 30, 1-9.

KeELE, S. W. Behavioral analysis of movement. In V. Brooks (Ed.), Handbook of physiology (Section 1) The nervous system (Vol. 2) Motor control (Part 2). Baltimore: Williams and Williams, 1981.

Kelso, J. A. S., Southard, D. L., \& Goodman, D. On the coordination of two-handed movements. Journal of Experimental Psychology: Human Perception and Performance, 1979, 5, 229-238.

KEPPEL, G. Design and analysis: $A$ researcher's handbook. Englewood Cliffs, N.J: Prentice-Hall, 1973.

KLA PP, S. T. Doing two things at once: The role of temporal compatibility. Memory \& Cognition, 1979, 7, 375-381.

MARTIN, J. G. Rhythmic (hierarchical) versus serial structure in speech and other behavior. Psychological Review, 1972, 79, 487-509.

Michon, J. A. Timing in temporal tracking. Assen, the Netherlands: Van Gorcum, 1967.

MontPEllie R, G. DE. Les alterations morphologiques des mouvements rapides. Louvain: Institut Superieur de Philosophie, 1935.

Perkins, D. N. Coding position in a sequence by rhythmic grouping. Memory \& Cognition, 1974, 2, 219-223.

Peters, M. Simultaneous performance of two motor activities: The factor of timing. Neuropsychologia, 1977, 15, 461-465.

Povel, D. J. Internal representation of simple temporal patterns. Journal of Experimental Psychology: Human Perception and Performance, 1981, 7, 3-18.

Shaffer, L. H. Performances of Chopin, Bach, and Bartok: Studies in motor programming. Cognitive Psychology, 1981, 13, 326-376.

Sturges, P. T., \& MARTIN, J. G. Rhythmic structure in auditory temporal pattern perception and immediate memory. Journal of Experimental Psychology, 1974, 102, 377-383.
Summers, J. J. The role of timing in motor program representation. Journal of Motor Behavior, 1975, 7, 229-241.

Treisman, M. Temporal discrimination and the indifference interval: Implications for a a model of the "internal clock." Psychological Monographs, 1963, 77(13, Whole No. 576).

WAGNER, C. The influence of the tempo of playing on the rhythmic structure studied at pianists playing scales. In E. Jokl \& H. Hebbelinck (Eds.), Medicine and sport VI. Basel, Switzerland: Karger, 1971.

WestgaARd, P. An introduction to tonal theory. New York: Norton, 1975.

Wing, A. M., \& Kristofferson, A. B. Response delays and the timing of discrete motor sequences. Perception \& Psychophysics, 1973, 14, 5-12. (a)

Wing, A. M., \& Kristofferson, A. B. The timing of interresponse intervals. Perception \& Psychophysics, 1973, 13, 455460. (b)

YESTON, M. The stratification of musical rhythm. New Haven: Yale University Press, 1976.

\section{NOTES}

1. The separate ear procedure was employed to maximize the chances of independent responding. If the two pulse trains had been presented to both ears simultaneously (for example, through a single loudspeaker), the development of an integrated pattern representation would have been encouraged.

2. The time window within which such a study can usefully be performed is quite narrow. With shorter base intervals, difficulties in following should arise at the shortest onset-to-onset interval. With longer base intervals, the impression of an integrated sequence should be strongly reduced at the longest onset-to-onset interval (Fraisse, 1982; Handel \& Oshinski, 1981).

3. See Keppel (1973), p. 139.

(Manuscript received February 7, 1983; revision accepted for publication June 14,1983 .) 